\title{
AN OVERVIEW OF HEALING OF TRAUMATIC TYMPANIC MEMBRANE PERFORATIONS
}

\author{
Parusharam Nagula1, Rajendra Prasad Janga ${ }^{2}$
}

${ }^{1}$ Associate Professor, Department of ENT, MGM Hospital, Warangal, Telangana, India.

${ }^{2}$ Senior Resident, Department of ENT, MGM Hospital, Warangal, Telangana, India.

ABSTRACT
BACKGROUND
Tympanic membrane traumatic perforation may be either due to direct or indirect source. The aim of the study is to evaluate the
various aetiologies of TM perforation at MGM Hospital, Warangal. Tympanic membrane is much more traumatized than middle or
inner ear. The incidence has been estimated at $6.80 / 1000$ persons. We wanted to evaluate the various aetiologies of TM
perforations, their prognosis and outcome with regard to various means (Conservative and Myringoplasty) of management.

\section{METHODS}

This is a retrospective descriptive study conducted from June 2015 to July 2017 in the Department of Otorhinolaryngology, KMC/MGM Hospital, Warangal, Telangana. The sample size was 50.

\section{RESULTS}

In this study, none of the patients got infected in the conservative treatment group. The average time taken for healing in uninfected cases (Conservative, Group 1) was around 64 days, whereas in Manipulated cases (Group 2, Myringoplasty) was 75 days. Improvement in hearing was determined by the audiometry report in both the groups, conservative treatment (15.5dB) and Myringoplasty group (19.3 dB).

\section{CONCLUSIONS}

Overall healing rates in both the conservative group as well as myringoplasty group were $100 \%$. There is no significant difference in the healing time taken for the tympanic membrane to heal completely in both the conservative as well myringoplasty group. Improvement in hearing as shown by the audiometry report in both the groups, conservative treatment and Myringoplasty group were comparable.

\section{KEY WORDS}

Perforation of Tympanic Membrane, Pure Tone Audiometry, Myringoplasty

HOW TO CITE THIS ARTICLE: Nagula P, Janga RP. An overview of healing of traumatic tympanic membrane perforations. J. Evolution Med. Dent. Sci. 2019;8(26):2126-2130, DOI: 10.14260/jemds/2019/467

\section{BACKGROUND}

TM, or eardrum, is a stiff but movable oval shaped membrane in the ear. It divides the outer ear canal from the middle ear. A perforation involves a tear or hole in the tympanic membrane. Tympanic membrane is much more traumatized than middle or inner ear. The incidence has been estimated at $6.80 / 1000$ persons. The cause of acute rupture of TM include direct trauma by instruments such as cotton swab, pins and sticks, iatrogenic such as syringing, suction and probing of ear and skull fracture. Pressure changes include blast injury and open palm trauma (Slapping), diving and flying.

As expected, the incidence of perforation of TM is on rise, consequent to increased violence and accidents seen in the society these days. Whether in war time or in peace time traumatic TM perforations have always been seen in otological trauma. The pathology of non-explosive blast injury is similar to explosive blast injury. However various

'Financial or Other Competing Interest': None.

Submission 07-12-2018, Peer Review 02-05-2019,

Acceptance 09-05-2019, Published 01-07-2019.

Corresponding Author:

Dr. Rajendra Prasad Janga,

H. No. 1-7-304, Pragathi Nagar,

Revenue Colony,

Hanmakonda-5060001,

Telangana, India.

E-mail: janga_janga98@yahoo.com

DOI: $10.14260 /$ jemds $/ 2019 / 467$

\section{(c) (i) $(9)$}

factors have proven to positively or negatively affect the individual susceptibility to TM rupture. The factors which include previous disease or injury, old age, inadequate pneumatization and position of TM perpendicular to incident wave, increase the chance of perforation. As a result of this perforation a conductive hearing loss may result. There may also be ear discharge, tinnitus (Ringing or buzzing sound in the ear), or dizziness. This discharge may be clear, bloody, or yellowish and thick Perforations may spontaneously heal within time but in the case of traumatic perforations a tympanoplasty may be performed to reduce the risk of infection. ${ }^{1}$ Injury to the tympanic membrane and the middle ear can be caused by over pressure from a slap on the ear, assault or road traffic injury. Thermal or caustic burns, penetration by instruments and barotraumas may also cause traumatic perforations. Perforations due thermal injury was caused by metal spark perforating the tympanic membrane during welding

Aim To find the incidence of a) Spontaneous healing of traumatic perforation of TM,b) Age and sex predilection. c) Associated hearing loss, type and severity of hearing loss. d) Tinnitus in patients with traumatic perforation e) Incidence of follow-up patients with hearing improvement and tinnitus resolution by conservative treatment alone.

TTMP patients normally suffers from reversible highfrequency hearing loss, usually complicated with tinnitus and vertigo. Most TTMPs (about 80\%) can heal spontaneously within 3 months post injury. However, large perforations 
usually fail to close, and appropriate treatment should be performed to reduce the incidence of permanent impairment.

The result of tympanic membrane perforation causes conductive hearing loss. This occurs because the advantages of ratio between the area of the tympanic membrane and the oval window is reduced, decreasing the vibrations transmitted (Gelfand, 2009). Consequently, the area to ratio difference between the tympanic membrane and oval window is also reduced. Whereas a fully intact membrane would give an approximate pressure gain at the oval window of times twenty, a perforated eardrum would not provide the same pressure gain. This means a significant amount of the impedance matching property of the middle ear is reduced. The malleus is attached to the tympanic membrane by the fibrous layer (Howard, 2009) and a perforation can affect this attachment by reducing the vibrations transmitted by the ossicular chain (Gelfand, 2009).2 Healing of the traumatic perforations of tympanic membrane should not be left to chance. A penetrating injury in the postero-superior quadrant may cause damage to the ossicular chain or a perilymphatic leak. Therefore, a careful middle ear examination is necessary to access the damage before starting the treatment. All most all studies says that $90 \%$ of traumatic perforations heal spontaneously within 90 days of injury. The patient besides symptomatic treatment need proper counseling and psychological support. Masterly inactivity should be religiously followed as spontaneous healing is achieved in more than $90 \%$ patients over a period of 2-14 weeks. People and health care professionals need to be educated and unskilled attempts at removal of wax and foreign bodies from external auditory canal need to be discouraged.

Traumatic perforation of TM is a common injury that is under-reported; there is the need to educate on unskilled removal of foreign body, early identification, evaluation and referral of patients so as to reduce the morbidity. Although traumatic TM perforations have good prognosis, it is necessary to induce patients with profuse explanations for possible complications to visit the out-patient clinic until the wound has healed completely.

Patching provides support to the healing epithelium of tympanic membrane, thus enhancing the healing process. It also protects the middle ear from any infection through the external auditory canal. In most studies, spontaneous healing of the tympanic membrane happens in about $80 \%$ of cases within 3 months of injury. ${ }^{1}$ Thus, masterly inactivity is the standard mode of treatment for the first 3 months. But in doing so, patients are unnecessarily exposed to disabling symptoms (decreased hearing, discharge from ears) and the need to modify lifestyles for those 3 months. If we can identify the causes of failure of spontaneous tympanic membrane healing after traumatic perforation, we may be able to recommend early intervention and reduce morbidity. This paper aims to study various aetiologies of traumatic tympanic membrane perforation; evaluate the factors involved in healing of traumatic tympanic membrane perforation; and identify the patients who are unlikely to benefit from conservative management so that early surgical intervention may be recommended.

\section{Aims and Objectives}

1. To evaluate the various aetiologies of traumatic tympanic membrane perforations.
2. To evaluate the prognosis and outcome of traumatic tympanic membrane perforations by various means of management.

\section{METHODS \\ Informed Consent}

Informed consent was obtained from all individual participants included in the study

\section{Study Design}

Retrospective Study.

\section{Study Period}

June 2015- July 2017.

\section{Place of Study}

Department of Otorhinolaryngology, MGM Hospital, Warangal, Telangana, India

\section{Sample Size}

50 .

\section{Inclusion Criteria}

1. Subjects 15-60 years of age and of both the sexes irrespective of socioeconomic status.

2. Able to comply with study procedures.

3. Informed written consent in English or Telugu.

\section{Exclusion Criteria}

1. Subjects underwent any form of ear surgery involving tympanic membrane in the past.

2. Subjects having middle ear infections.

\section{Methodology}

After obtaining clearance and approval from the Institutional Ethics Committee, 50 patients fulfilling Inclusion/Exclusion who give Informed Consent will be included in the study.

Data is collected from the patients presenting to the ENT department. Following data will be collected from case sheets of patients in a Study Proforma.

And outcome was measured based on the average time taken for healing, the improvement in hearing as shown by the audiometry report in both the groups.

\section{Statistical Analysis}

Quantitative or qualitative results will be analysed using Zscore or chi square test wherever applicable.

\section{RESULTS}

Out of 50 patients, 30 patients(Group 1) were directly came to hospital without any manipulation, and remaining 20 patients (Group 2) were presented to hospital after few days to weeks with some manipulation (Local ear drops, oil, powder, ear buds and etc.). In this study, none of the patients got infected in the conservative treatment group. Average time taken for healing by the uninfected Cases (Conservative, Group 1) was 64 days, whereas in Manipulated cases (Group 2, Myringoplasty) was 75 days. It can be shown the improvement in hearing as shown by the audiometry report in both the groups, conservative treatment $(15.5 \mathrm{~dB})$ and Myringoplasty group (19.3 dB). 

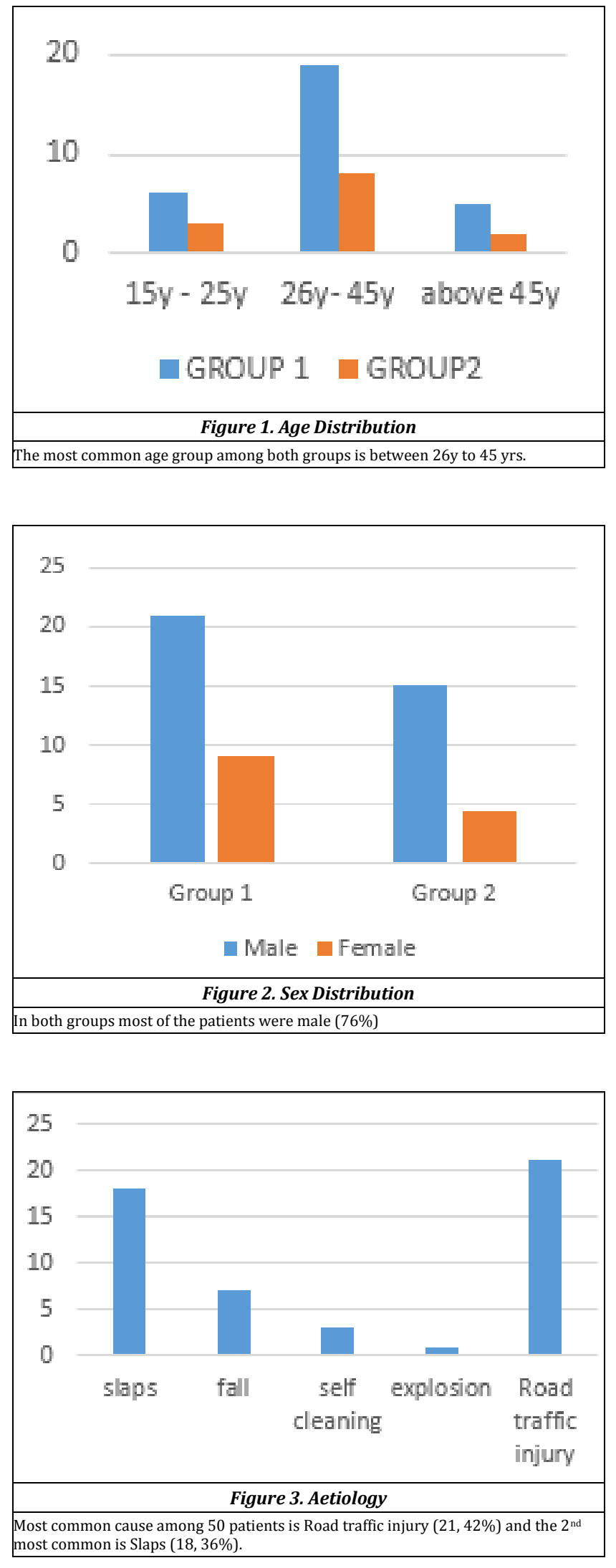
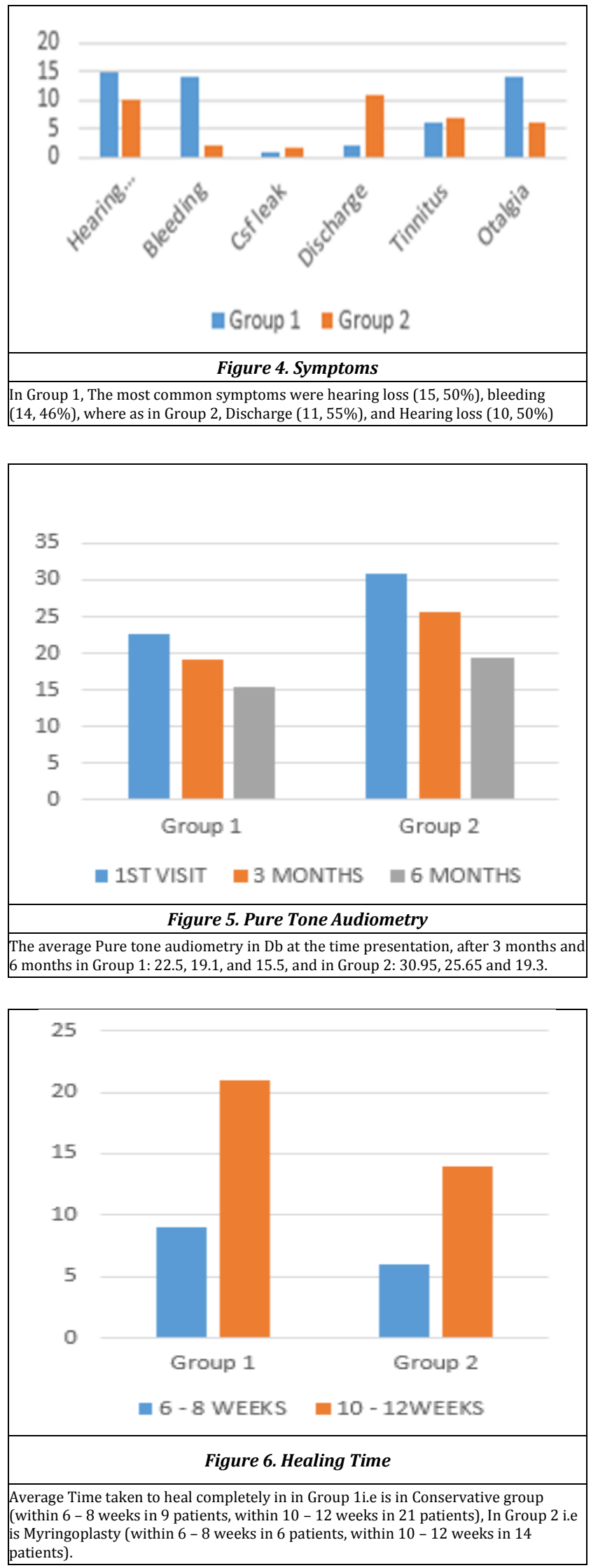

\section{DISCUSSION}

The normal tympanic membrane is a translucent, graycoloured, lustrous, concave, and oval-shaped structure separating outer ear from the middle ear. It is divided into pars tensa and pars flaccida by anterior and posterior malleolar folds. Tympanic membrane conducts sound waves 
across the middle ear and protects the middle ear cleft from infection.

Tympanic membrane perforation occurs when there is a break in its continuity, resulting in a hole with direct communication between the external auditory canal and the middle ear cleft. This increases the potential to middle ear cleft infection with a resultant hearing impairment.

Injury to the ear could be a simple blunt trauma to the pinna or laceration of the pinna or avulsion of a part of the pinna or the whole pinna, uncomplicated TMP, ossicles damage and petrous temporal bone longitudinal and transverse fractures with associated loss of inner ear and facial nerve function.

TM play a vital role in sound conduction as its vibratory characteristic is necessary for sound transmission in humans ${ }^{3}$. Trauma to TM can be caused by over pressure, blunt trauma or penetrating injuries and barotraumas ${ }^{4,5}$. In this study, Road traffic injury was the most common cause of trauma to TM similar to various studies elsewhere. Traumatic TM perforations are seen in all age groups. In our study, middle age group (26-45 years) has the highest incidence similar to studies undertaken by Gacek and Gacek and Berger et $\mathrm{al}^{6}$ There is no sex predominance. Lindeman et al. ${ }^{7}$ Reported greater prevalence among females similar to study reported by Lou et al. ${ }^{8}$ However, a higher male involvement was reported by Gacek and Gacek and da Lilly-Tariah and Somefun. The higher prevalence among males. In our study, $100 \%$ of patients had unilateral involvement. Right ear involvement was seen in $26.85 \%$ of patients and left ear was involved in $71.10 \%$ patients. This could be associated with the fact that most assailants were right handed and likely that most of the acts of trauma such as slap occurred with the assailant and the victim facing each other making the left ear more vulnerable to trauma. Lindeman et al. and Berger et al. reported a similar predilection for left ear. Attempts at removal of foreign bodies from external auditory canal, ear cleaning with a various type of objects and wax removal in an unskilled manner either by parents, quacks or primary care physician are other main causes of trauma as are also reported in various other studies. That's why there is a need for the primary care physician to draw the red lines and routinely consider the referral in all such cases. In our study regardless of mechanism of injury, Hearing loss was the most common complaint (50\%) in Group 1. but the common complaint was discharge from ear in Group 2. In the study by Berger et al and da Lilly-Tariah and Somefun, hearing loss followed by tinnitus and otalgia were common complaints. In this study, hearing loss increased with increase in size of perforation at each frequency. It is due to hydraulic action arising from the difference in area of foot plate and TM, the main factor in impedance matching. If the surface area is decreased, there is decrease in amplification and hearing loss will be proportional to the size of perforation. Most traumatic perforation have a tendency to heal spontaneously, there was $100 \%$ healing in our study similar to other studies 9-12. Residual perforation was observed only in $0 \%$ patients. The data strongly suggests that prolonged observation remains an excellent option for patients presenting with traumatic TM perforation. Traumatic perforations often occur in community and generally the prognosis is excellent. The two main factors that predispose to failure of perforation to heal area, loss of tissue, and secondary infection.
Perforation causes hearing loss, which depends on frequency, perforation size and middle-ear space. Our study confirmed that with the increase in perforation size hearing loss also increases, with the lowest frequencies being affected more often; this is consistent with other studies in the literature and the study by Santhi and Rajan. Who in their study found that perforation-induced losses are greatest at the lowest frequencies and that larger perforations result in larger hearing losses.

\section{CONCLUSIONS}

In traumatic TM perforation, early surgical intervention is not indicated as chances of spontaneous healing are very high. Overall healing rates in both the conservative group as well as myringoplasty group were $100 \%$. There was no significant difference in the healing time taken for TM to heal completely in both the conservative as well myringoplasty group. In our study, it can be concluded that improvement in hearing, as shown by the audiometry report in both the groups, conservative treatment and myringoplasty group were comparable.

\section{REFERENCES}

[1] Amadasun JE. An observational study of the management of traumatic tympanic membrane perforations. The Journal of Laryngology \& Otology 2002;116(3):181-4.

[2] Gelfand SA. Essentials of audiology. New York, USA: Thieme Medical Publishers Inc., 2009: p. 171-2.

[3] Gacek RR, Gacek MR. Anatomy of the auditory and vestibular systems. In: Snow JB Jr, Ballenger JJ, eds. Ballenger's Otorhinolaryngology Head and Neck Surgery. Vol. 1. 16 $6^{\text {th }}$ edn. Ontario: DC Becker Inc, 2003: p. 1-5.

[4] Mitchell SK. Trauma to the middle ear, inner ear, and temporal bone. In: Snow JB Jr, Ballenger JJ, eds. Ballenger's Otorhinolaryngology Head and Neck Surgery. Vol. 14. $16^{\text {th }}$ edn. DC Becker Inc, 2003: p. 34556.

[5] Da Lilly-Tariah OB, Somefun AO. Traumatic perforation of the tympanic membrane in University of Port Harcourt Teaching Hospital, Port Harcourt. Nigeria. Niger Postgrad Med J 2007;14(2):121-4.

[6] Berger G, Finkelstein Y, Harell M. Non-explosive blast injury of the ear. J Laryngol Otol 1994;108(5):395-8.

[7] Lindeman P, Edström S, Granström G, et al. Acute traumatic tympanic membrane perforations. Cover or observe? Arch Otolaryngol Head Neck Surg 1987;113(12):1285-7.

[8] Lou ZC, Lou ZH, Zhang QP. Traumatic tympanic membrane perforations: a study of aetiology and factors affecting outcome. Am J Otolaryngol 2012;33(5):549-55.

[9] Orji FT, Agu CC. Determinants of spontaneous healing in traumatic perforations of the tympanic membrane. Clin Otolaryngol 2008;33(5):420-6.

[10] Kristensen S, Juul A, Gammelgaard NP, et al. Traumatic tympanic membrane perforations: complications and management. Ear Nose Throat J 1989;68(7):503-16. 


\section{Jemds.com}

[11] Yamazaki K, Ishijima K, Sato H. A clinical study of traumatic tympanic membrane perforation. Nihon Jibiinkoka Gakkai Kaiho 2010;113(8):679-86.
Original Research Article

[12] Ijaduola GT. The principles of management of deafness. Niger Med Pract 1986;12:19-25. 